\title{
Two-dimensional folding technique for enhancing Fermi surface signatures in the momentum density: Application to Compton scattering data from an Al-3 at. \% Li disordered alloy
}

\author{
I. Matsumoto \\ Department of Synchrotron Radiation Science, The Graduate University for Advanced Studies, Tsukuba, Ibaraki 305-0801, Japan \\ J. Kwiatkowska and F. Maniawski \\ H. Niewodniczanski Institute of Nuclear Physics, Radzikowskiego 152, 31-342 Cracow, Poland
}

M. Itou

Spring-8, Kamigouri, Akougun, Hyogo 679-5198, Japan

H. Kawata

Photon Factory, High Energy Accelerator Research Organization, Tsukuba, Ibaraki 305-0801, Japan

N. Shiotani

Tokyo University of Fisheries, Kounan, Minato, Tokyo 108-8477, Japan

S. Kaprzyk

Department of Physics, Northeastern University, Boston, Massachusetts 02115

and Academy of Mining and Metallurgy, Al. Mickiewicza 30, Cracow, Poland

P.E. Mijnarends

Department of Physics, Northeastern University, Boston, Massachusetts 02115

and Interfaculty Reactor Institute, Delft University of Technology, Delft, The Netherlands

B. Barbiellini and A. Bansil

Department of Physics, Northeastern University, Boston, Massachusetts 02115

(Received 12 February 2001; published 9 July 2001)

\begin{abstract}
We present a technique for enhancing Fermi surface (FS) signatures in the two-dimensional (2D) distribution obtained after the 3D momentum density in a crystal is projected along a specific direction in momentum space. These results are useful for investigating fermiology via high-resolution Compton scattering and positron annihilation spectroscopies. We focus on the particular case of the (110) projection in a fcc crystal where the standard approach based on the use of the Lock-Crisp-West (LCW) folding theorem fails to give a clear FS image due to the strong overlap with FS images obtained through projection from higher Brillouin zones. We show how these superposed FS images can be disentangled by using a selected set of reciprocal lattice vectors in the folding process. The applicability of our partial folding scheme is illustrated by considering Compton spectra from an $\mathrm{Al}-3$ at. \% Li disordered alloy single crystal. For this purpose, high-resolution Compton profiles along nine directions in the (110) plane were measured. Corresponding highly accurate theoretical profiles in Al-3 at. \% Li were computed within the local density approximation (LDA)-based Korringa-KohnRostoker coherent potential approximation (KKR-CPA) first-principles framework. A good level of overall accord between theory and experiment is obtained, some expected discrepancies reflecting electron correlation effects notwithstanding, and the partial folding scheme is shown to yield a clear FS image in the (110) plane in $\mathrm{Al}-3$ at. $\% \mathrm{Li}$.
\end{abstract}

DOI: $10.1103 /$ PhysRevB.64.045121

PACS number(s): 71.18.+y, 78.70.Ck, 41.60.Ap, 78.70.Bj

\section{INTRODUCTION}

Recent advances in synchrotron light sources and detector technology have rejuvenated interest in high-resolution Compton scattering as a probe of fermiology and electron correlation effects in wide classes of materials. ${ }^{1-14}$ In a Compton scattering experiment one measures a directional Compton profile (CP), $J\left(p_{z}\right)$, which is related to the twiceintegrated ground-state electron momentum density $\rho(\mathbf{p})$ by

$$
J\left(p_{z}\right)=\iint \rho(\mathbf{p}) d p_{x} d p_{y},
$$

where $p_{z}$ is taken along the scattering vector of the $\mathrm{x}$ rays. In the independent particle approximation,

$$
\rho(\mathbf{p})=\sum_{b, \mathbf{k}}\left|\int \psi_{b, \mathbf{k}}(\mathbf{r}) e^{-i \mathbf{p} \cdot \mathbf{r}} d \mathbf{r}\right|^{2},
$$

where $\psi_{b, \mathbf{k}}(\mathbf{r})$ is the electron wave function in band $b$ and state k. The summation in Eq. (1.2) extends over all initial states that are excited in the scattering process. Throughout this paper this difinition of $\Sigma_{b, \mathbf{k}}$ is used. The CP thus con- 
tains signatures of the Fermi surface (FS) breaks and correlation effects in the underlying three-dimensional (3D) momentum density $\rho(\mathbf{p})$.

The determination of FS geometry via the Compton spectroscopy is complicated by the presence of the double integral in Eq. (1.1). As a result, FS breaks in $\rho(\mathbf{p})$ do not in general induce rapid variations in $J\left(p_{z}\right)$, but only in the first derivative of the profile. Moreover, breaks in $\rho(\mathbf{p})$ caused by FS crossings are scattered throughout momentum space with weights given by the appropriate matrix element. Therefore, if this matrix element happens to be small in a specific case, ${ }^{15}$ then the related FS feature in the $\mathrm{CP}$ will be intrinsically weak and difficult to measure. The standard approach to tackle these problems is to measure CP's along many different directions, and to invert Eq. (1.1) to obtain $\rho(\mathbf{p})$ by using one of the available reconstruction methods. ${ }^{\rho, 17}$ The matrix element effects in $\rho(\mathbf{p})$ can then be removed in principle by constructing the occupation number density in $\mathbf{p}$ space, $N(\mathbf{p})$, via the so-called Lock-Crisp-West (LCW) folding procedure: ${ }^{18}$

$$
N(\mathbf{p}) \equiv \sum_{\mathbf{G}} \rho(\mathbf{p}+\mathbf{G})=\sum_{\mathbf{G}} \sum_{b, \mathbf{k}} \delta_{\mathbf{k}, \mathbf{p}+\mathbf{G}}
$$

where the sum over $\mathbf{G}$ runs over all reciprocal lattice vectors. The right hand side of Eq. (1.3) represents the occupation number density in the repeated zone scheme. ${ }^{19}$

In practical applications, an accurate reconstruction of $\rho$ (p) along the preceding lines requires the demanding task of making CP measurements along a suitable group of directions. However, in many cases the $2 \mathrm{D}$ projection of the momentum density, $\rho\left(p_{x}, p_{y}\right)=\int \rho(\mathbf{p}) d p_{z}$, and the corresponding $2 \mathrm{D}$ occupation number density, $N\left(p_{x}, p_{y}\right)$, along a judiciously chosen direction can give most of the important features of the FS. As shown in Sec. II it is then sufficient to measure a set of CP's with scattering vectors all lying in the plane of projection, yielding a significant reduction of measuring time. The (110) plane in cubic lattices encompasses many of the high symmetry points and lines in the Brillouin zone and, therefore, a cross section in this plane captures much of the character of the underlying 3D Fermi surface. In this vein, the (110) projection has been exploited in a number of Compton scattering and positron annihilation investigations ${ }^{20-23}$ on bcc crystals where the (110) projection turns out to be reasonably free of complications of overlapping FS images from higher Brillouin zones. In sharp contrast, it has not been possible to use this approach in fcc crystals due to overlap problems that often distort the FS image severely.

With this motivation, we have examined the question of how the aforementioned problems of overlap may be avoided in a projected 2D momentum distribution. Our analysis reveals that the problem can be solved by using a selected subset of reciprocal vectors in the folding procedure of Eq. (1.3). Although we focus in this article explicitly on the specific case of the (110) projection in an fcc crystal, these ideas are generalized straightforwardly to other projections in cubic lattices. The treatment of projections in general lattices will be taken up elsewhere.
We illustrate the applicability of our folding procedure by considering Compton spectra from an $\mathrm{Al}-3$ at. \% Li disordered alloy (fcc) single crystal specimen. For this purpose, nine high-resolution CP's have been measured along a set of directions in the (110) plane, and corresponding highly accurate theoretical profiles have been computed within the KKR-CPA first-principles framework. Intercomparisons between theory and experiment, and between results based on the use of a selected and a full set of reciprocal lattice vectors in the folding process, clearly show the value of our partial folding approach in enhancing FS signatures in the data.

Some comment on our choice of the $\mathrm{Al}-3$ at. \% Li disordered alloy for this study is appropriate. $\mathrm{Al}$, which possesses an almost spherical FS (in the extended zone), is an interesting base metal for exploring the evolution of fermiology with changes in the electrons/atom ratio via alloying on the one hand, and for investigating electron correlation effects on the momentum density in multivalent systems on the other. Metallurgically, $\mathrm{Al}$ is quite "exclusive" in that only a few percent impurities can be dissolved in the solid solution $\alpha$ phase. ${ }^{24}$ For example, in Al-Li the $\alpha$ phase extends at room temperature to only about 3 at. \% $\mathrm{Li}$ concentration. Al-Li alloys, with the possible addition of other elements, are also of great technological interest due to their high strength-toweight ratio. ${ }^{25}$ For these fundamental and practical reasons, the electronic structure and the nature of bonding in $\mathrm{Al}-\mathrm{Li}$ alloys have drawn considerable interest. ${ }^{26-28}$ Insofar as the Compton scattering technique is concerned, relatively little by the way of high-resolution work on disordered alloys is available in the literature despite the fact that, unlike traditional spectroscopies which rely on quantum oscillatory phenomena, Compton scattering is not affected by short electron mean free paths and is thus particularly suited for investigating disordered alloys. For reasons outlined in this paragraph, there is a strong motivation for undertaking a state-of-the-art Compton scattering study of the Al-Li system as a means of gaining insight into the electronic structure of the system and the possible role of fermiology in destabilizing the $\alpha$ phase of $\mathrm{Al}^{29}$

This article is organized as follows. Section II briefly delineates some reconstruction aspects needed later. It is shown that it suffices to measure a set of CP's with scattering vectors all lying in one plane to obtain the $2 \mathrm{D}$ projection of the momentum density onto that plane. In Sec. III, the folding procedure involving selected reciprocal lattice vectors is formulated. Section IV gives pertinent details of the Compton scattering experiments, while Sec. V briefly describes the relevant computational details involved in obtaining theoretical momentum densities and Compton profiles. Section VI presents and discusses the theoretical and experimental Compton scattering results as well as the applicability of our partial folding procedure to these data. Some concluding remarks are collected in Sec. VII.

\section{RECONSTRUCTION}

The 3D momentum density can be reconstructed by using the Fourier-Bessel method in which a so-called $B(\mathbf{r})$ function is defined by 


$$
B(\mathbf{r})=\int \rho(\mathbf{p}) \exp (-i \mathbf{p} \cdot \mathbf{r}) d \mathbf{p} .
$$

It follows from Eqs. (1.1) and (2.1) that

$$
B(0,0, z)=\int J\left(p_{z}\right) \exp \left(-i p_{z} z\right) d p_{z} .
$$

Thus, from a number of measured CP's, corresponding $B(\mathbf{r})$ 's are obtained on radii in $\mathbf{r}$ space given by the directions of the respective scattering vectors. Next, a fine mesh is set up in $\mathbf{r}$ space and $B(\mathbf{r})$ is obtained at every mesh point by interpolation. This makes it then possible to carry out the inverse Fourier transform

$$
\rho(\mathbf{p})=(2 \pi)^{-3} \int B(\mathbf{r}) \exp (i \mathbf{p} \cdot \mathbf{r}) d \mathbf{r}
$$

to obtain $\rho(\mathbf{p})$. We refer the reader to Ref. 17 for a detailed discussion of our reconstruction scheme.

Here we briefly show how a 2D projection of the momentum density along a given direction can be reconstructed from Compton scattering measurements in which all the scattering vectors lie in the perpendicular plane. Without loss of generality, we take [001] to be the projection direction. From the inverse Fourier transform equation (2.3) the projection onto the (001) plane is

$$
\begin{aligned}
\rho\left(p_{x}, p_{y}\right)= & \int \rho(\mathbf{p}) d p_{z}=(2 \pi)^{-3} \int d p_{z} \iiint B(x, y, z) \\
& \times \exp \left[i\left(p_{x} x+p_{y} y+p_{z} z\right)\right] d x d y d z
\end{aligned}
$$

The integration with respect to $p_{z}$ produces $2 \pi \delta(z)$, yielding

$$
\rho\left(p_{x}, p_{y}\right)=(2 \pi)^{-2} \iint B(x, y, 0) \exp \left[i\left(p_{x} x+p_{y} y\right)\right] d x d y .
$$

But, as seen from Eq. (2.2) above, $B(x, y, 0)$ can be obtained by interpolation on a fine mesh in the $(x, y)$ plane from measurements in which all scattering vectors lie in that plane.

\section{FORMULATION OF THE PARTIAL FOLDING TECHNIQUE}

This section delineates briefly how the (110) projection of the LCW-folded momentum density in Eq. (1.3) can be divided for an fcc crystal into two identical patterns where one pattern is shifted by $2 \pi / a$ along the [001] direction with respect to the other. We consider an fcc lattice with primitive translation vectors:

$$
\mathbf{a}=\frac{a}{2}(\hat{\mathbf{x}}+\hat{\mathbf{y}}), \quad \mathbf{b}=\frac{a}{2}(\hat{\mathbf{y}}+\hat{\mathbf{z}}), \quad \mathbf{c}=\frac{a}{2}(\hat{\mathbf{z}}+\hat{\mathbf{x}})
$$

where $\hat{\mathbf{x}}, \hat{\mathbf{y}}, \hat{\mathbf{z}}$ are unit vectors along the Cartesian axes. A general lattice vector is

$$
\mathbf{R}=u \mathbf{a}+v \mathbf{b}+w \mathbf{c}, \quad u, v, w \text { are integers. }
$$

Similarly, the primitive translation vectors of the (bcc) reciprocal lattice are

$$
\mathbf{A}=\frac{2 \pi}{a}(\hat{\mathbf{x}}+\hat{\mathbf{y}}-\hat{\mathbf{z}}), \quad \mathbf{B}=\frac{2 \pi}{a}(-\hat{\mathbf{x}}+\hat{\mathbf{y}}+\hat{\mathbf{z}}),
$$

$$
\mathbf{C}=\frac{2 \pi}{a}(\hat{\mathbf{x}}-\hat{\mathbf{y}}+\hat{\mathbf{z}}),
$$

and the general reciprocal lattice vector is

$$
\mathbf{G}=h \mathbf{A}+k \mathbf{B}+l \mathbf{C}, \quad h, k, l \text { are integers. }
$$

Because we are interested specifically in projecting onto the (110) plane, it is convenient to work with new unit vectors $\hat{\mathbf{s}}$ and $\hat{\mathbf{t}}$ that lie along and perpendicular to [110]:

$$
\hat{\mathbf{s}}=\frac{1}{\sqrt{2}}(\hat{\mathbf{x}}+\hat{\mathbf{y}}), \quad \hat{\mathbf{t}}=\frac{1}{\sqrt{2}}(\hat{\mathbf{x}}-\hat{\mathbf{y}}),
$$

and thus

$$
\mathbf{R}=R_{s} \hat{\mathbf{s}}+R_{t} \hat{\mathbf{t}}+R_{z} \hat{\mathbf{z}}
$$

with

$$
\begin{gathered}
R_{s}=\frac{a}{2 \sqrt{2}}(2 u+v+w), \quad R_{t}=\frac{a}{2 \sqrt{2}}(-v+w), \\
R_{z}=\frac{a}{2}(v+w),
\end{gathered}
$$

while

$$
\mathbf{G}=G_{s} \hat{\mathbf{s}}+G_{t} \hat{\mathbf{t}}+G_{z} \hat{\mathbf{z}}
$$

with

$$
\begin{gathered}
G_{s}=\frac{2 \pi}{a} \sqrt{2} h, \quad G_{t}=\frac{2 \pi}{a} \sqrt{2}(-k+l), \\
G_{z}=\frac{2 \pi}{a}(-h+k+l) .
\end{gathered}
$$

Likewise, we have

$$
\mathbf{p}=p_{s} \hat{\mathbf{s}}+p_{t} \hat{\mathbf{t}}+p_{z} \hat{\mathbf{z}}, \quad \mathbf{r}=r_{s} \hat{\mathbf{s}}+r_{t} \hat{\mathbf{t}}+r_{z} \hat{\mathbf{z}} .
$$

The momentum density projected onto the (110) plane is

$$
\int \rho(\mathbf{p}) d p_{s}=\rho\left(p_{t}, p_{z}\right) .
$$

Having established the relevant notation, we write the momentum density Eq. (1.2) as

$$
\begin{aligned}
\rho(\mathbf{p}) & =\rho\left(p_{s}, p_{t}, p_{z}\right) \\
& =\sum_{b, \mathbf{k}} \iint_{\text {crystal }} d \mathbf{r} d \mathbf{r}^{\prime} \psi_{b, \mathbf{k}}(\mathbf{r}) \psi_{b, \mathbf{k}}^{*}\left(\mathbf{r}^{\prime}\right) e^{-i \mathbf{p} \cdot\left(\mathbf{r}-\mathbf{r}^{\prime}\right)} .
\end{aligned}
$$

Writing the wave functions in the Bloch form and converting the integral over $\mathbf{r}$ in Eq. (1.2) into a sum of integrals over a single cell, one obtains 


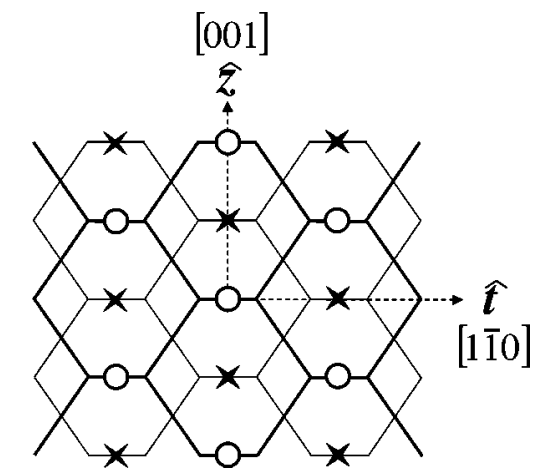

FIG. 1. The bcc reciprocal lattice points (corresponding to an fcc crystal) are shown projected onto the (110) plane. $\hat{\mathbf{z}}$ denotes the [001] and $\hat{\mathbf{t}}$ the [1 $\overline{1} 0]$ direction. As discussed in the text, open circles refer to points with even $h$ in Eq. (3.8) and crosses to points with odd $h$. Outlines of the Brillouin zones for the sublattices of circles and crosses are shown by thin and thick solid lines, respectively.

$$
\begin{aligned}
\rho\left(p_{t}, p_{z}\right)= & \int d p_{s} \sum_{b, \mathbf{k}} \sum_{\mathbf{R}, \mathbf{R}^{\prime}} \iint d \mathbf{r} d \mathbf{r}^{\prime} u_{b, \mathbf{k}}(\mathbf{r}) u_{b, \mathbf{k}}^{*}\left(\mathbf{r}^{\prime}\right) \\
& \times e^{i \mathbf{k} \cdot(\mathbf{R}+\mathbf{r})} e^{-i \mathbf{k} \cdot\left(\mathbf{R}^{\prime}+\mathbf{r}^{\prime}\right)} e^{-i \mathbf{p} \cdot\left(\mathbf{R}-\mathbf{R}^{\prime}\right)} e^{-i \mathbf{p} \cdot\left(\mathbf{r}-\mathbf{r}^{\prime}\right)} .
\end{aligned}
$$

The two-dimensional projection of the occupation number density $N\left(p_{t}, p_{z}\right)$ obtained by LCW folding over all projected reciprocal lattice vectors is

$$
N\left(p_{t}, p_{z}\right)=\sum_{G_{t}, G_{z}} \rho\left(p_{t}+G_{t}, p_{z}+G_{z}\right)
$$

where the sum runs over all sets $\left(G_{t}, G_{z}\right)$.

The formulation so far leading to Eq. (3.12) has been quite general. The nature of the problem as well as its solution may be delineated with reference to Fig. 1, which shows schematically the projection of the bcc reciprocal lattice onto the (110) plane. The lattice points in the (110) plane going through the origin [for which $h=0$ in Eq. (3.8)] lie at the positions of the circles. The two nearest (110) planes $(h$ $= \pm 1$ ) lie at a distance of $G_{s}=(2 \pi / a) \sqrt{2}$ in front of and behind this central plane and the corresponding lattice points project into the crosses. The next pair of planes projects to the circles and then the process repeats itself. The entire bcc reciprocal lattice is thus divided into two sublattices related to the circles and the crosses with associated 2D Brillouin zones shown by light and dark lines respectively. ${ }^{30}$ It is easily seen now that when the images of the Fermi surface given by the periodic LCW-folded 3D density are projected along [110], the contributions from the circled and crossed sublattices will overlap and get entangled. However, since the circled and crossed sublattices are related by a simple shift of $2 \pi / a$ along the $z$ axis, if the contribution of one of these sublattices can be suppressed in the folding procedure, the projection will no longer suffer from the aforementioned complication.

The preceding strategy may be carried out more formally as follows. In the $(s, t, z)$ space, Eqs. (3.8) give the coordi- nates of various bcc lattice points generated via the integers $h, k$, and $l$. Different layers perpendicular to the [110] direction correspond to different values of $h$. It is easily shown then that the circled sublattice in Fig. 1 involves only even values of the index $h$ where $G_{t}$ and $G_{z}$ [ignoring prefactors in Eqs. (3.8)] are either both even or both odd. Similarly, the crossed sublattice is given by odd values of $h$ where $G_{t}$ and $G_{z}$ (again, ignoring prefactors) possess opposite parities.

Bearing these considerations in mind, we divide the summation in Eq. (3.12) into two sums involving even and odd values of $h$ and we use Eq. (3.11). This yields

$$
\begin{aligned}
N\left(p_{t}, p_{z}\right)= & \sum_{G_{t}, G_{z}}^{h \text { even }} \rho\left(p_{t}+G_{t}, p_{z}+G_{z}\right) \\
& +\sum_{G_{t}, G_{z}}^{h \text { odd }} \rho\left(p_{t}+G_{t}, p_{z}+G_{z}\right) \\
= & \sum_{G_{t}, G_{z}} \sum_{b, \mathbf{k}} \sum_{\mathbf{R}, \mathbf{R}^{\prime}} \int d p_{s} \iint d \mathbf{r} d \mathbf{r}^{\prime} u_{b, \mathbf{k}}(\mathbf{r}) u_{b, \mathbf{k}}^{*}\left(\mathbf{r}^{\prime}\right) \\
& \times e^{i \mathbf{k} \cdot(\mathbf{R}+\mathbf{r})} e^{-i \mathbf{k} \cdot\left(\mathbf{R}^{\prime}+\mathbf{r}^{\prime}\right)} e^{-i p_{s}\left(R_{s}-R_{s}^{\prime}+r_{s}-r_{s}^{\prime}\right)} \\
& \times e^{-i\left(p_{t}+G_{t}\right)\left(R_{t}-R_{t}^{\prime}+r_{t}-r_{t}^{\prime}\right)} e^{-i\left(p_{z}+G_{z}\right)\left(R_{z}-R_{z}^{\prime}+r_{z}-r_{z}^{\prime}\right)} \\
& +(\text { a similar expression with } h \text { odd }) .
\end{aligned}
$$

We now make use of the following identities:

$$
\begin{gathered}
\int d p_{s} e^{-i p_{s}\left(R_{s}-R_{s}^{\prime}+r_{s}-r_{s}^{\prime}\right)}=\delta_{R_{s}^{\prime} R_{s}} \delta\left(r_{s}-r_{s}^{\prime}\right), \\
\sum_{G_{t}, G_{z}} e^{-i G_{t}\left(r_{t}-r_{t}^{\prime}\right)-i G_{z}\left(r_{z}-r_{z}^{\prime}\right)}=\delta\left(r_{t}-r_{t}^{\prime}\right) \delta\left(r_{z}-r_{z}^{\prime}\right),
\end{gathered}
$$

$$
\sum_{R_{t}} \sum_{R_{z}} e^{i\left(k_{t}-p_{t}\right) R_{t}} e^{i\left(k_{z}-p_{z}\right) R_{z}}=\delta\left(k_{t}, p_{t}+G_{t}^{a}\right) \delta\left(k_{z}, p_{z}+G_{z}^{a}\right),
$$

where for the sake of simplicity we ignore forefactors, $\delta(a, b)$ denotes the Kronecker delta $\delta_{a, b}$, and $\left(G_{t}^{a}, G_{z}^{a}\right)$ are the components of an arbitrary reciprocal lattice vector $\mathbf{G}^{\mathbf{a}} \cdot{ }^{31}$ Furthermore,

$$
e^{-i G_{t}\left(R_{t}-R_{t}^{\prime}\right)-i G_{z}\left(R_{z}-R_{z}^{\prime}\right)}= \begin{cases}1 & \text { for even } h \\ e^{-(2 \pi / a) i\left(R_{z}-R_{z}^{\prime}\right)} & \text { for odd } h\end{cases}
$$

The last identity is easily seen by using Eqs. (3.6) and (3.8) and realizing that $v+w=(2 / a) R_{z}$. The final result is

$$
N^{\text {even }}\left(p_{t}, p_{z}\right)=c \sum_{G_{t}^{e}, G_{z}^{e}}^{h \text { even }} \sum_{b, \mathbf{k}} \delta\left(k_{t}, p_{t}+G_{t}^{e}\right) \delta\left(k_{z}, p_{z}+G_{z}^{e}\right)
$$

and 


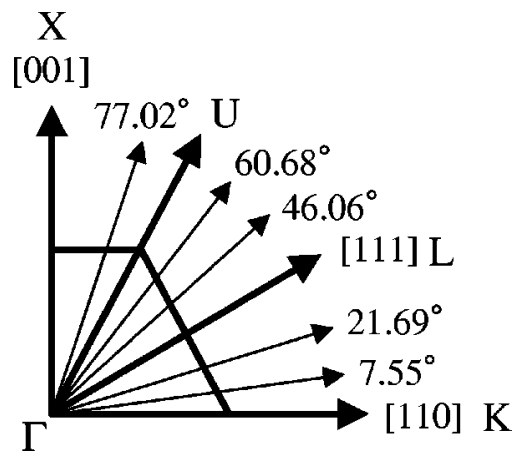

FIG. 2. The nine directions in the (110) plane along which the Compton profiles were measured and computed in this study.

$$
\begin{aligned}
N^{o d d}\left(p_{t}, p_{z}\right)= & c \sum_{G_{t}^{o}, G_{z}^{o}}^{h \text { odd }} \sum_{b, \mathbf{k}} \delta\left(k_{t}, p_{t}+G_{t}^{o}\right) \\
& \times \delta\left(k_{z}, p_{z}+\frac{2 \pi}{a}+G_{z}^{o}\right),
\end{aligned}
$$

where the constant $c$ collects the irrelevant prefactors and $G_{t}^{e(o)}$ and $G_{z}^{e(o)}$ are the components of $\mathbf{G}$ with even (odd) $h .^{32}$

The right-hand side of Eq. (3.18) is just the projected two-dimensional $\mathbf{k}$ space occupation sampling function in the repeated zone scheme for the set of reciprocal lattice vectors with even $h$ in Eq. (3.3), while the right-hand side of Eq. (3.19) fulfills the same function for the set with odd $h$. Thus, the set of Fermi surface images appearing in Eq. (3.18), reappear in Eq. (3.19) but shifted by $2 \pi / a$ along $\hat{\mathbf{z}}$. By carrying out the LCW folding over only one of these two sets of reciprocal lattice vectors, the Fermi surfaces corresponding to the other set are removed and thus a picture is obtained which is not complicated by superposition.

Since $N^{\text {even }}$ and $N^{\text {odd }}$ are identical (apart from the shift) and their sum yields the occupation function $N\left(p_{t}, p_{z}\right)$, it follows that $N^{\text {even }}$ and $N^{\text {odd }}$ each carry half the intensity of the occupation function. Finally, it is clear that the present procedure can be used straightforwardly in the analysis of positron annihilation 2D ACAR spectra from an fcc crystal.

\section{EXPERIMENT}

A single crystal of $\mathrm{Al}-3$ at. \% Li was grown from the melt by the Bridgman-Stockbarger method under a pressurized argon atmosphere to reduce loss of Li. The composition was determined by both atomic absorption spectrometry and proton-induced gamma emission and the final specimen, 10 $\mathrm{mm}$ in diameter and $1.5 \mathrm{~mm}$ thick, was found to contain 3.0 at. \% Li. The Compton profiles along nine directions, all lying in the (110) plane (see Fig. 2), were measured with the KEK-AR spectrometer. ${ }^{33}$ The incident photon energy was 60 $\mathrm{keV}$, which is high enough so that the impulse approximation would be satisfied quite well. The overall momentum resolution in the measurements is estimated to be 0.12 a.u. The total number of accumulated counts under the profiles was about $1 \times 10^{8}$. The necessary energy-dependent corrections for absorption, detector, and analyzer efficiency and scattering cross section were applied. The contribution of double scattering was simulated via the Monte Carlo program of Sakai. ${ }^{34}$ The integrated intensity of the double scattering events was found to be $10 \%$ of that of the single scattering events. By using Eq. (2.2), the nine measured profiles yield the values of $B(z)$ along nine radial directions all of which lie in the (110) plane in real space. $B(\mathbf{r})$ was then interpolated over an area of $50 \times 50$ a.u. ${ }^{2}$ on a fine square mesh of $500 \times 500 \mathbf{r}$ points in the $(110)$ plane. The projection $\rho\left(p_{t}, p_{z}\right)$ of the 3D momentum density was obtained via an inverse 2D Fourier transformation of the interpolated $B(\mathbf{r})$. The $\rho\left(p_{t}, p_{z}\right)$ thus obtained was partially folded following the procedure of Sec. III to obtain the $2 \mathrm{D}$ occupation number density.

\section{COMPUTATIONS}

The computation of the electronic structure of $\mathrm{Al}-3$ at. \% $\mathrm{Li}$ was carried out within the all-electron charge selfconsistent KKR-CPA framework and is parameter-free. A lattice constant of $a=7.6534$ a.u. was assumed. The underlying KKR-CPA methodology is described in Refs. 35-39. The relevant Green's function formulation for treating the momentum density and Compton profile in disordered alloys is given in Refs. 40 and 41. Exchange and correlation effects were incorporated within the von Barth-Hedin local density approximation. ${ }^{42,43}$ In order to obtain the Compton profile the momentum density $\rho(\mathbf{p})$ was first evaluated over a fine mesh of $48 \times 4851 \times 1777 \mathbf{p}$ points covering momenta up to $p_{\max } \sim 5$ a.u. Compton profiles with scattering vectors along the same directions as the experimental ones were then computed accurately by evaluating the integral of Eq. (1.1). The accuracy of the computed profiles is about 1 part in $10^{4}$. The nine theoretical profiles were convoluted with the experimental resolution and then treated in a manner identical to that of the nine experimental profiles.

\section{APPLICATION TO AL-3 AT. \% LI ALLOY}

We briefly compare first the theoretical and experimental directional profiles by taking the [100] CP as an example; results along other directions are similar. After subtraction of the core contribution the two profiles of Fig. 3 are normalized by area to yield the same number of electrons over the range \pm 4 a.u. The theoretical $\mathrm{CP}$ is seen to be higher than the experimental one at low momenta with the situation reversing itself at high momenta. The Fermi cutoff in the first derivative of the $\mathrm{CP}$ around 1 a.u. is sharper in the theory; this effect is also evident in the second derivative where the corresponding calculated peak is higher and narrower compared to the measurements even though the theoretical CP's and their derivatives include resolution broadening. Similar discrepancies between theory and experiment have been observed previously in other systems and can be ascribed to the failure of the present LDA-based independent particle model to properly account for correlations in the electron gas. ${ }^{5-13}$ These correlation effects result in the shift of spectral weight from below to above the Fermi momentum $\left(p_{F}\right)$ and a reduction in the size of the break $\left(Z_{k}\right)$ in the momentum den- 

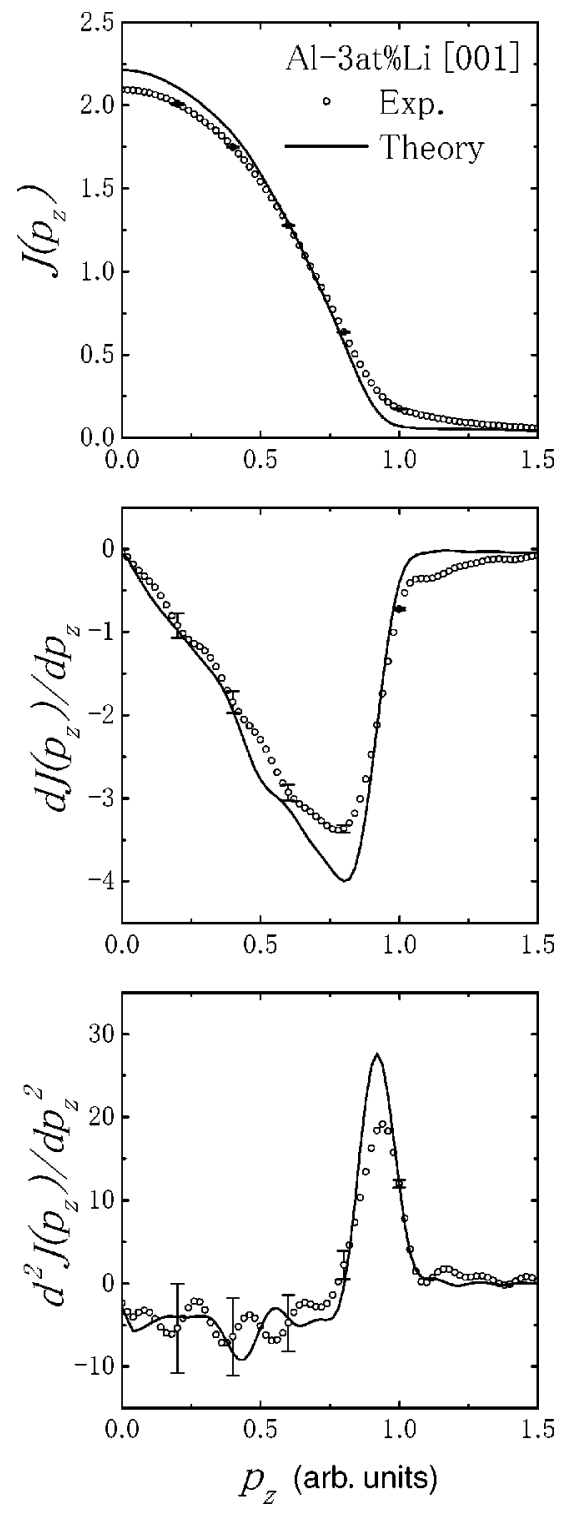

FIG. 3. Experimental and theoretical directional Compton profiles in $\mathrm{Al}-3$ at. \% Li and the associated first and second derivatives are compared along the [001] direction. The theoretical results have been broadened to reflect the experimental resolution.

sity at $p_{F}$. Concerning fine structure, features around 0.3 a.u. and 0.6 a.u. in the first derivative of the experimental $\mathrm{CP}$ are reproduced reasonably by theory. These features are quite similar to those found in $\mathrm{Al}$ in the positron annihilation (1D angular correlation) experiments ${ }^{44}$ as well as in the recent high-resolution Compton scattering measurements. ${ }^{45}$ In the case of Al, these have been explained by the fact that the Fermi surface of $\mathrm{Al}$ deviates from a sphere (in the extended zone) and bulges on the hexagonal zone face near the $\mathrm{W}-\mathrm{K}-\mathrm{W}$ and $\mathrm{W}-\mathrm{U}-\mathrm{W}$ zone edges; ${ }^{46}$ this mechanism is presumably at play in $\mathrm{Al}-3$ at. \% $\mathrm{Li}$ as well since the addition of 3 at. \% Li induces relatively little change in the Fermi surface of $\mathrm{Al}$.

Before discussing the effects of folding, it is helpful to consider Fig. 4, which shows contour maps of the reconstructed experimental and theoretical momentum density in
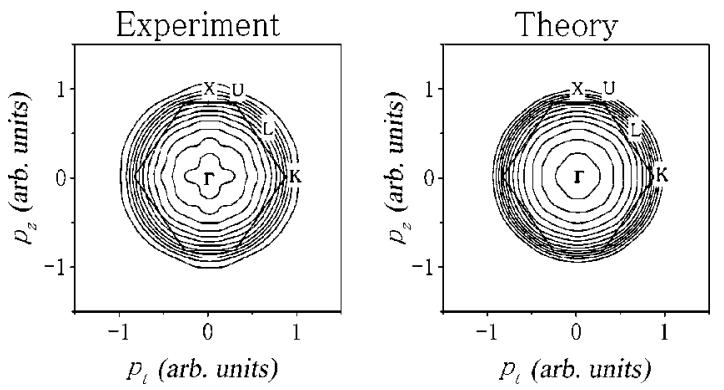

FIG. 4. Contour maps of the reconstructed experimental and theoretical momentum densities in the $(110)$ or $\left(p_{z}, p_{t}\right)$ plane. Note that the results shown represent the 2D-distribution obtained after the $3 \mathrm{D}$ momentum density is integrated along the [110] direction. The first Brillouin zone boundary and the high symmetry points in the (110) plane are indicated.

the (110) plane. We emphasize that an integration along [110] (i.e., $p_{s}$ ) is implicit here as well as throughout this article. Therefore, what is shown in all cases is the (110) 2D projection of the underlying 3D momentum density distribution. As expected, the data of Fig. 4 display twofold crystalline symmetry, but it is difficult to ascertain the details of the FS, except perhaps that it is roughly spherical in shape (in the extended zone) with some bulging along the $\langle 110\rangle$ directions.

Insight into the nature of our partial folding scheme is provided by Fig. 5, which considers the case of the freeelectron model where the Fermi sphere corresponds to 2.94 electrons/atom appropriate to the number of conduction electrons in $\mathrm{Al}-3$ at. \% Li. Figure 5(a) has been obtained via the conventional LCW-folding procedure in which all reciprocal lattice vectors are used, whereas Fig. 5(b) includes the contribution of only one of the sublattices as discussed in Sec. III. The imprint of the free-electron FS (repeated periodically) is obvious in the 2D occupation number density of Fig. 5(b), even though this is far from being the case in Fig. 5(a), (a)

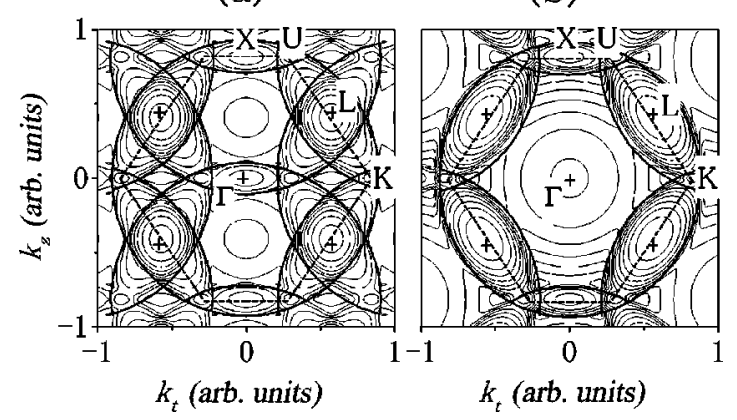

FIG. 5. Contour maps of the $2 \mathrm{D}$ occupation number density (after projection along [110]), $N\left(k_{z}, k_{t}\right)$, in the (110) plane for the free-electron model at the electron concentration of $\mathrm{Al}-3$ at. \% $\mathrm{Li}$. (a) Conventional LCW folding using all reciprocal lattice vectors. (b) Present partial LCW folding scheme using selected reciprocal lattice vectors. Experimental resolution broadening is included in the calculations. The Brillouin zone boundary and the projected free-electron sphere are shown. + signs indicate high-density regions. 
(a) Theory

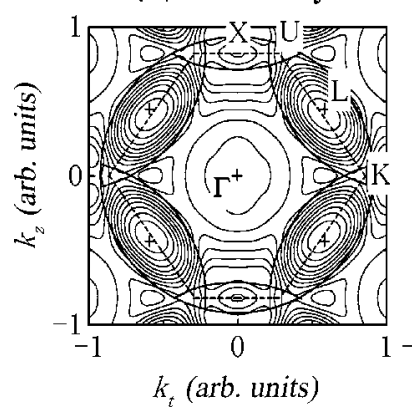

(b) Experiment

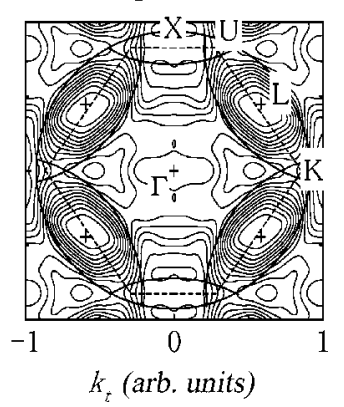

FIG. 6. Contour maps of the theoretical and experimental 2D occupation number densities (after projection along [110]), $N\left(k_{z}, k_{t}\right)$, in the (110) plane based on the present partial LCWfolding scheme in Al-3 at. \% Li. Resolution broadening is included in the theory. See caption to Fig. 5 for other notational details.

which shows the appearance of four crosslike features around the $L$ symmetry points due to overlapping Fermi surfaces. Furthermore, complicated structures arise in Fig. 5(a) around the zone center. It is striking how the two overlapping Fermi surfaces in Fig. 5(a) are disentangled in Fig. 5(b). ${ }^{47}$ These results show clearly the power of the present partial folding approach for analyzing FS images in terms of the (110) projection in an fcc crystal.

The application to $\mathrm{Al}-3$ at. \% $\mathrm{Li}$ is considered in Fig. 6, which compares theoretical and experimental (110)projected, partially folded, 2D occupation densities. On the whole, the FS contours in Fig. 6 are rather like those in Fig. 5 (b), indicating that the FS of the alloy is more or less spherical, despite some deviations. Note that the FS contours in Fig. 6 are not symmetrical with respect to the $U-K$ lines, even though this is strictly true for the free-electron case in Fig. 5(b). This is a consequence of the asphericity of the FS; the FS bulges more outside the hexagonal zone face near the $W-K-W$ zone edge (where two hexagonal faces of the first zone intersect) than near the $W-U-W$ zone edge (intersection between a hexagonal and a square face of the first zone). This can also be described as a slight flattening of the FS in the vicinity of $U$, combined with a small expansion near $K$. Theory and experiment are in good overall accord in Fig. 6, although the fact that the oblong maximum around the $L$ point in Fig. 6(b) is slightly more rotated compared to Fig. 6(a) suggests that the aforementioned asphericity of the FS is somewhat stronger in the experiment.

Finally, we consider radial cuts through the 2D momentum distribution of Fig. 4; a typical cut (along $\Gamma-L$ ) is shown in Fig. 7. It is well known that the value of the Fermi momentum $p_{F}$ is not given correctly by the inflection point in the profile of Fig. 7(a), or equivalently, the position of the minimum in the first derivative of Fig. 7(b). ${ }^{48}$ The situation may be simulated by considering the free-electron case. Here the $2 \mathrm{D}$ distribution corresponding to the profile of Fig. 7(a) is a semicircle and the first derivative is negative and diverges at $p_{F}$. When the semicircle is convoluted with the experimental resolution, a tail appears beyond $p_{F}$ and the divergence of the first derivative at $p_{F}$ disappears. The inflection point defining the minimum of the first derivative then
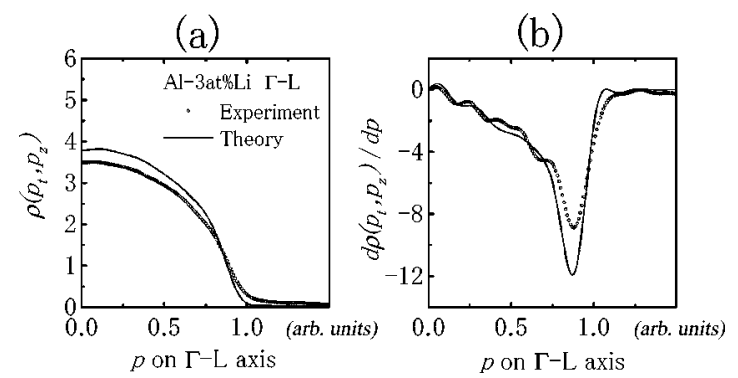

FIG. 7. Radial sections along the $\Gamma-L$ direction through the experimental and theoretical [110]-projected momentum distributions of Fig. 4 are shown together with the corresponding first derivatives.

moves below $p_{F}$ to an extent which depends on the width of the resolution function. Accordingly, by using a free-electron semicircle of radius 0.919 a.u. and the present experimental resolution of 0.12 a.u., we have determined that the position of the inflection points in cuts such as those of Fig. 7 must be increased by $4.2 \%$ in order to obtain the correct value of $p_{F}$. Applying this correction, theoretical and experimental $p_{F}$ values in the (110) plane can be mapped out from the data of Fig. 4. The results of the analysis are given in Fig. 8, and show an excellent level of accord between theory and experiment. Nevertheless, a careful examination of Fig. 8 reveals a slight contraction of the experimental FS (compared to theory) near $U$ in combination with a slight expansion near $K$, consistent with our earlier discussion in connection with Fig. 6 above.

\section{SUMMARY AND CONCLUSIONS}

We address the question of how Fermi surface (FS) signatures can be enhanced in the 2D distribution obtained by projecting the 3D momentum density along the [110] direction in an fcc crystal. The standard LCW folding procedure invoked in this connection involves a summation of the momentum density over all reciprocal lattice vectors to obtain the electron occupation number density in the system. This procedure has been used to produce a reasonably clear FS imprint, even when the (110)-projected momentum density is considered, in several studies of bcc crystals, but it has not been successful in fcc crystals. We show, however, that the

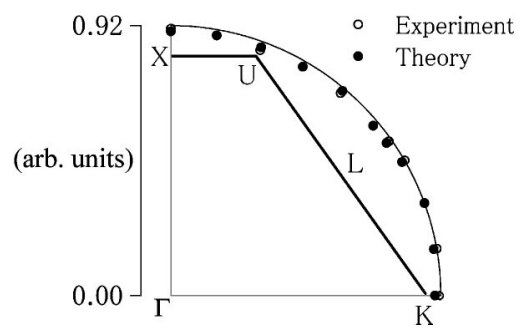

FIG. 8. Fermi momentum $p_{F}$ in the (110) plane in the $\mathrm{Al}-3$ at. \% Li disordered alloy. Open circles give results deduced from the present Compton profile measurements, while the KKR-CPAbased theoretical predictions are given by filled circles. The solid circular arc indicates the free-electron momentum of 0.919 a.u. for Al-3 at. \% Li. 
(110) projection of the LCW-folded momentum density in the fcc lattice can be viewed as a superposition of two equivalent FS images that are shifted by $2 \pi / a$ along the [001] axis, and that these two images can be disentangled if one uses a selected subset of reciprocal lattice vectors in the folding process. Our folding procedure is particularly well suited for investigating fermiology-related issues via high resolution Compton experiments where a full 3D reconstruction of the data is far more demanding than a $2 \mathrm{D}$ reconstruction using measured directional profiles, all of which involve scattering vectors in the (110) plane. The technique will also be valuable in analyzing positron annihilation (2D ACAR) experiments in fcc lattices.

An extensive application of the aforementioned partial folding procedure to the case of an fcc disordered alloy is presented. To this end, we have measured nine highresolution directional Compton profiles on a single-crystal specimen of $\mathrm{Al}-3$ at. \% $\mathrm{Li}$ with scattering vectors in the (110) plane. We have also carried out highly accurate computations of corresponding theoretical profiles within the self-consistent, parameter-free KKR-CPA framework. Occupation number densities based on theoretical as well as the experimental profiles obtained via partial folding display clear images of the FS. A reasonable level of accord is found between theory and experiment with respect to the overall shape of the directional profiles as well as the fine structure in the first and the second derivatives. The experimental results with regard to the FS are also in accord with KKR-CPA predictions, although there is an indication of a slightly stronger bulging of the measured Fermi surface outside the hexagonal zone face along the $W-K-W$ than along the $W-U$ $W$ zone edge. Upon adding 3 at. \% $\mathrm{Li}$, the $\mathrm{FS}$ of $\mathrm{Al}$ remains essentially free-electron-like, some overall shrinking of its size due to the reduced $e / a$ ratio notwithstanding. Large FS sheets in $\mathrm{Al}$ thus show no sign of an anomaly that might destabilize the $\alpha$ phase. A Fermi surface-driven mechanism in this connection will therefore need to focus on small features in the $\mathrm{FS}$ of $\mathrm{Al}$ (electron pockets in the third conduction band) that can undergo substantial change and may even disappear with decreasing electron concentration or altered electron lattice interaction upon alloying.

\section{ACKNOWLEDGMENTS}

The Compton profile measurements were performed with the approval of the Photon Factory Advisory Committee, Proposal No. 97G288. This work is supported by the Polish Committee for Scientific Research, Grant No. 2 P03B 028 14, the U.S. Department of Energy Contract No. W-31-109ENG-38, and benefited from the allocation of supercomputer time at NERSC and the Northeastern University Advanced Scientific Computation Center (ASCC).
${ }^{1}$ M.J. Cooper, Rep. Prog. Phys. 48, 415 (1985).

${ }^{2}$ A. Bansil, Z. Naturforsch., A: Phys. Sci. 48, 165 (1993).

${ }^{3}$ P.E. Mijnarends and A. Bansil, in Positron Spectroscopy of Sol$i d s$, edited by A. Dupasquier and A.P. Mills, Jr. (IOS, Amsterdam, 1995), p. 25.

${ }^{4}$ S. Manninen, J. Phys. Chem. Solids 61, 335 (2000).

${ }^{5}$ Y. Sakurai, Y. Tanaka, A. Bansil, S. Kaprzyk, A.T. Stewart, Y. Nagashima, T. Hyodo, S. Nanao, H. Kawata, and N. Shiotani, Phys. Rev. Lett. 74, 2252 (1995).

${ }^{6}$ W. Schülke, G. Stutz, F. Wohlert, and A. Kaprolat, Phys. Rev. B 54, 14381 (1996).

${ }^{7}$ K. Hämäläinen, S. Manninen, C.-C. Kao, W. Caliebe, J.B. Hastings, A. Bansil, S. Kaprzyk, and P.M. Platzman, Phys. Rev. B 54, 5453 (1996).

${ }^{8}$ M. Itou, Y. Sakurai, T. Ohata, A. Bansil, S. Kaprzyk, Y. Tanaka, H. Kawata, and N. Shiotani, J. Phys. Chem. Solids 59, 99 (1998).

${ }^{9}$ S. Huotari, K. Hämäläinen, S. Manninen, S. Kaprzyk, A. Bansil, W. Caliebe, T. Buslaps, V. Honkimäki, and P. Suortti, Phys. Rev. B 62, 7956 (2000).

${ }^{10}$ N. Shiotani, Y. Tanaka, Y. Sakurai, N. Sakai, M. Ito, F. Itoh, T. Iwazumi, and H. Kawata, J. Phys. Soc. Jpn. 62, 239 (1993).

${ }^{11}$ Y. Sakurai, S. Kaprzyk, A. Bansil, Y. Tanaka, G. Stutz, H. Kawata, and N. Shiotani, J. Phys. Chem. Solids 60, 905 (1999).

${ }^{12}$ G. Stutz, F. Wohlert, A. Kaprolat, W. Schülke, Y. Sakurai, Y. Tanaka, M. Ito, H. Kawata, N. Shiotani, S. Kaprzyk, and A. Bansil, Phys. Rev. B 60, 7099 (1999).

${ }^{13}$ P. Suortti, T. Buslaps, V. Honkimäki, C. Metz, A. Shukla, Th. Tschentscher, J. Kwiatkowska, F. Maniawski, A. Bansil, S.
Kaprzyk, A.S. Kheifets, D.R. Lun, T. Sattler, J.R. Schneider, and F. Bell, J. Phys. Chem. Solids 61, 397 (2000).

${ }^{14}$ I. Matsumoto, J. Kwiatkowska, F. Maniawski, A. Bansil, S. Kaprzyk, M. Itou, H. Kawata, and N. Shiotani, J. Phys. Chem. Solids 61, 375 (2000).

${ }^{15}$ R. Harthoorn and P.E. Mijnarends, J. Phys. F: Met. Phys. 8, 1147 (1978).

${ }^{16}$ See, e.g., Refs. 12 and 17 for recent studies of reconstructed momentum densities based on high-resolution Compton data.

${ }^{17}$ Y. Tanaka, Y. Sakurai, A.T. Stewart, N. Shiotani, P.E. Mijnarends, S. Kaprzyk, and A. Bansil, Phys. Rev. B 63, 045120 (2001).

${ }^{18}$ D.G. Lock, V.H.C. Crisp, and R.N. West, J. Phys. F: Met. Phys. 3, 561 (1973).

${ }^{19}$ The form on the right-hand side of Eq. (1.3) is of course based on the independent particle model and thus assumes the absence of correlations inherent therein.

${ }^{20}$ S.B. Dugdale, H.M. Fretwell, K.J. Chen, Y. Tanaka, A. Shukla, T. Buslaps, Ch. Bellin, G. Loupias, M.A. Alam, A.A. Manuel, P. Suortti, and N. Shiotani, J. Phys. Chem. Solids 61, 361 (2000).

${ }^{21}$ Y. Tanaka, K.J. Chen, C. Bellin, G. Loupias, H.M. Fretwell, A. Rodriguez-Gonzalez, M.A. Alam, S.B. Dugdale, A.A. Manuel, A. Shukla, T. Buslaps, P. Suortti, and N. Shiotani, J. Phys. Chem. Solids 61, 365 (2000).

${ }^{22}$ H.M. Fretwell, S.B. Dugdale, A. Rodriguez-Gonzalez, M.A. Alam, N. Shiotani, M.J. Cooper, V. Sundararajan, and R.M. Singru, Mater. Sci. Forum 255-257, 417 (1997).

${ }^{23}$ C.R. Bull, A. Alam, R.N. West, N. Shiotani, A.K. Singh, and R.M. Singru, in Positron Annihilation, edited by P.C. Jain, R.M. 
Singru, and K.P. Gopinathan (World Scientific, Singapore 1985), p. 266.

${ }^{24}$ See, for example, Binary Alloy Phase Diagrams, edited by T.B. Massalski (ASM International, 1990).

${ }^{25}$ E.J. Lavernia and N.J. Grant, J. Mater. Sci. 22, 1521 (1987).

${ }^{26}$ A. Zunger, Phys. Rev. B 17, 2582 (1978).

${ }^{27}$ X.-Q. Guo, R. Podloucky, and A.J. Freeman, Phys. Rev. B 42, 10912 (1990).

${ }^{28}$ V. Kumar, Phys. Rev. B 60, 2916 (1999).

${ }^{29} \mathrm{~A}$ high-resolution Compton study of the [111] profile in $\mathrm{Al}-3$ at. \% $\mathrm{Li}$ is reported in Ref. 14.

${ }^{30}$ This partitioning does not correspond to the more common one where the bcc lattice is viewed as a superposition of two simple cubic lattices shifted by half the cube diagonal with respect to each other.

${ }^{31}$ In evaluating Eq. (3.16) it should be noted that the values of $R_{t}$ and $R_{z}$ in the summations are not independent but coupled by the symmetry of the direct lattice, expressed in Eq. (3.1). Thus, both $\left(k_{t}-p_{t}\right) R_{t}=2 n \pi$ and $\left(k_{t}-p_{t}\right) R_{t}=(2 n+1) \pi$ lead to admissable solutions (namely $G_{t}, G_{z}$ both even and both odd, respectively, for even $h$ ) since in the second case the minus sign of the $z$ factor cancels that of the $t$ factor.

${ }^{32}$ In a similar manner, by multiplying the left side of Eq. (3.17) by $\exp \left[2 \pi i\left(v-v^{\prime}\right)\right]=1$ and noting that $-v+w=(2 \sqrt{2} / a) R_{t}$, it can be shown that the two lattices differ by a shift of $2 \pi \sqrt{2} / a$ along $\hat{\mathbf{t}}$.

${ }^{33}$ Y. Sakurai, M. Ito, T. Urai, Y. Tanaka, N. Sakai, T. Iwazumi, H. Kawata, M. Ando, and N. Shiotani, Rev. Sci. Instrum. 63, 1190 (1992).

${ }^{34}$ N. Sakai, J. Phys. Soc. Jpn. 56, 2477 (1987).
${ }^{35}$ A. Bansil, S. Kaprzyk, and J. Tobola, in Applications of Multiple Scattering Theory to Materials Science, edited by W.H. Butler, P.H. Dedericks, A. Gonis, and R. Weaver, Mater. Res. Soc. Symp. Proc. No. 253, (Materials Research Society, Warrendale, PA, 1992), p. 505.

${ }^{36}$ A. Bansil, S. Kaprzyk, P.E. Mijnarends, and J. Tobola, Phys. Rev. B 60, 13396 (1999).

${ }^{37}$ S. Kaprzyk and A. Bansil, Phys. Rev. B 42, 7358 (1990).

${ }^{38}$ A. Bansil and S. Kaprzyk, Phys. Rev. B 43, 10335 (1991).

${ }^{39}$ S. Kaprzyk, Acta Phys. Pol. A 91, 135 (1997).

${ }^{40}$ P.E. Mijnarends and A. Bansil, Phys. Rev. B 13, 2381 (1976).

${ }^{41}$ A. Bansil, R.S. Rao, P.E. Mijnarends, and L. Schwartz, Phys. Rev. B 23, 3608 (1981).

${ }^{42}$ U. von Barth and L. Hedin, J. Phys. C 5, 1629 (1972); A.K. Rajagopal and J. Callaway, Phys. Rev. B 7, 1912 (1973).

${ }^{43}$ Other, perhaps more satisfactory, forms of the exchangecorrelation functional could of course be used in the process of obtaining the selfconsistent crystal potential. However, the effects are not particularly significant, especially within the context of this article.

${ }^{44}$ T. Okada, H. Sekizawa, and N. Shiotani, J. Phys. Soc. Jpn. 41, 836 (1976).

${ }^{45}$ T. Ohata, M. Itou, I. Matsumoto, Y. Sakurai, H. Kawata, N. Shiotani, S. Kaprzyk, P.E. Mijnarends, and A. Bansil, Phys. Rev. B 62, 16528 (2000).

${ }^{46}$ Y. Kubo, S. Wakoh, and J. Yamashita, J. Phys. Soc. Jpn. 41, 830 (1976).

${ }^{47}$ Of course, the remaining overlap in Fig. 5(b) is due to the size of the Fermi surfaces centered on neighboring $\Gamma$ points and is inherent in the electronic structure.

${ }^{48}$ For a recent discussion see, e.g., Ref. 17. 